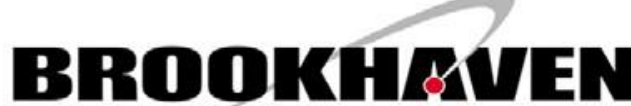 \\ NATIONAL LABORATORY
}

BNL-114436-2017-JA

\section{Gas transport selectivity of ultrathin, nanoporous, inorganic membranes made from block copolymer templates}

\author{
S. Greil, M. Liu \\ Submitted to the Journal American Chemical Society \\ October 2017 \\ Center for Functional Nanomaterials \\ Brookhaven National Laboratory

\section{U.S. Department of Energy USDOE Office of Science (SC), Basic Energy Sciences (BES) (SC-22)}

\footnotetext{
Notice: This manuscript has been authored by employees of Brookhaven Science Associates, LLC under Contract No. DE- SC0012704 with the U.S. Department of Energy. The publisher by accepting the manuscript for publication acknowledges that the United States Government retains a non-exclusive, paid-up, irrevocable, world-wide license to publish or reproduce the published form of this manuscript, or allow others to do so, for United States Government purposes.
} 


\section{DISCLAIMER}

This report was prepared as an account of work sponsored by an agency of the United States Government. Neither the United States Government nor any agency thereof, nor any of their employees, nor any of their contractors, subcontractors, or their employees, makes any warranty, express or implied, or assumes any legal liability or responsibility for the accuracy, completeness, or any third party's use or the results of such use of any information, apparatus, product, or process disclosed, or represents that its use would not infringe privately owned rights. Reference herein to any specific commercial product, process, or service by trade name, trademark, manufacturer, or otherwise, does not necessarily constitute or imply its endorsement, recommendation, or favoring by the United States Government or any agency thereof or its contractors or subcontractors. The views and opinions of authors expressed herein do not necessarily state or reflect those of the United States Government or any agency thereof. 


\section{Gas transport selectivity of ultrathin, nanoporous, inorganic membranes made from block copolymer}

\section{templates}

Stefanie Greil, Atikur Rahman ${ }^{\dagger}$, Mingzhao Liu*, and Charles T. Black*

Center for Functional Nanomaterials, Brookhaven National Laboratory, Upton, NY 11973, USA

ABSTRACT

We report the fabrication of ultrathin, nanoporous silicon nitride membranes made from templates of regular, nanoscale features in self-assembled block copolymer thin films. The inorganic membranes feature thicknesses less than $50 \mathrm{~nm}$ and volume porosities over 30 percent, with straight-through pores that offer high throughout for gas transport and separation applications. As fabricated, the pores are uniformly around $20 \mathrm{~nm}$ in diameter, but can be controllably and continuously tuned to single-digit nanometer dimensions by combining with conformal coatings by atomic layer deposition. A deviation from expected Knudsen diffusion is revealed for transport characteristics of saturated vapors of organic solvents across the membrane, which becomes more significant for membranes of smaller pores. We attribute this to capillary condensation of saturated vapors within membrane pores, which reduces membrane throughput by over one order of magnitude but significantly improves the membrane's 
selectivity. Between vapors of acetone and ethyl acetate, we measure selectivities as high as 7:1 at ambient pressure and temperature, four times more than the Knudsen selectivity.

Membranes are used to separate two continuous phases and restrict the exchange of chemical species between the two phases. ${ }^{1}$ Selective membranes are integral to modern society, with widespread application in batteries, water filtration, industrial chemical synthesis, and separation of liquids and gases..$^{2-7}$ In fluid separation applications, the two key membrane performance criteria are selectivity between those material designed to be transported and those to inhibit, and the overall membrane throughput. For any membrane design, optimizing one criteria compromises the other, so that tradeoffs are typically made between the two. Selective membranes may have either atomically dense (non-porous) structures or porous structures that feature interconnected microscopic or mesoscopic pores. ${ }^{1}$ These two types of membranes follow very different separation principles, and have nearly opposite separation characteristics. Nonporous membranes separate materials based on their different solubility within the membrane and, usually enjoy high selectivity at the cost of low throughput. On the other hand, porous membranes separate materials by discriminating between molecular sizes, and generally feature high throughput with relatively low selectivity.

Porous selective membranes are most commonly fabricated from ceramic or glassy materials, including alumina, titania, zirconia, and silicon carbide, using fabrication techniques such as sol-gel coating, controlled pyrolysis, and chemical vapor deposition. ${ }^{8-12}$ For mechanical stability, the porous membrane is typically supported on a macroporous substrate. ${ }^{12}$ Given the bottom-up nature for most of the fabrication techniques, the pores formed within the membrane have relatively random size, shape, and topology. Rather than connecting both sides of the membrane, a significant portion of the pores can be isolated or dead-ended, thus making no contribution to the separation process. ${ }^{13}$ Here we report a combined bottom-up and top-down 
technique that uses self-assembled block copolymer thin films to template fabricate ultrathin, inorganic membranes with predetermined pore density and dimensions for gas/vapor separations.

Block copolymers spontaneously form periodic patterns of phase separated nanodomains, with the lattice types and parameters determined by the block copolymer molecular weights and chemical composition. ${ }^{14-16}$ Self-assembled patterns in block copolymer thin films can be transferred to fabricate patterned nanostructures/devices, in a process analogous to lithographic patterning. ${ }^{15-19}$ Selective removal of one copolymer phase creates a porous structure that has intrinsically high porosity and can be made ultrathin - both of which aid high membrane throughput. Previously, nanoporous polymeric membranes derived from block copolymers have been considered and demonstrated as useful for applications such as gas separation, water ultrafiltration, and drug delivery. ${ }^{20-28}$ These studies have generally used organic/polymeric porous films, made by selectively removing one of the copolymer blocks. To date, although block copolymer lithography has been widely used to fabricate nanostructured thin films on supported substrates, there are few demonstrations of suspended membranes by this technique. In this work, we demonstrate that the block copolymer nanopattern can be transferred to inorganic materials for mechanically robust, suspended ultrathin membranes, which provide durability desired for operation at high temperatures or in extreme environments, and with the ability to be chemically functionalized for further improved selectivity. We further demonstrate that, although the as-fabricated inorganic membranes offer limited gas separation selectivity due to the relatively large pore size $(10-20 \mathrm{~nm})$, improvements are readily made by uniformly and controllably shrinking the pores through ALD.

We create inorganic membranes less than $50 \mathrm{~nm}$ thick with 20 to 30 percent porosity using a fabrication process combining block copolymer self-assembly and pattern transfer through plasma etching (Figure 1a). Our substrate is a $100 \mathrm{~nm}$ thick suspended silicon nitride window with area $88 \times 74 \mu^{2}$, supported on a $3 \times 3 \mathrm{~mm}^{2}$ silicon substrate (SPI Supplies, Inc.). Onto 
these substrates a thin film of polystyrene-block-poly(methylmethacrylate) (PS- $b$-PMMA) is coated, which self-assembles into an ordered cylindrical phase morphology of hexagonally arranged PS cylinders embedded in a PMMA matrix via self-assembly (Figure 1b). The morphology is determined by the block copolymer molecular weight $\left(71 \mathrm{~kg} \mathrm{~mol}^{-1}\right)$ and its chemical composition (30:70 PS:PMMA by weight) - the complement to the PMMA cylinder phase typically employed when using PS- $b$-PMMA block copolymers. ${ }^{16,29,30}$ The copolymer molecular weight dictates the pattern's average cylinder diameters of $d=30 \pm 3 \mathrm{~nm}$, with $\ell=42$ $\pm 5 \mathrm{~nm}$ nearest neighbor separation (center-to-center).

We replicate the features of the self-assembled block copolymer film in the underlying silicon nitride window through a series of two pattern transfer steps, resulting in a porous structure with pore sizes and density mimicking those of the initial template. Prior to pattern transfer to the silicon nitride, we first selectively infuse PMMA regions of the self-assembled polymer film with alumina through sequential exposures to vapor pressures of trimethylaluminum and water vapor. ${ }^{31,32}$ Subsequent removal of the block copolymer using an oxygen plasma leaves a porous alumina film that replicates the morphology of the PMMA regions, atop the silicon nitride membrane (Figure 1c). This structure provides a rugged mask for further transfer of the pattern's nanoscale features into and through the silicon nitride using an inductively coupled plasma etch (Figure 1d). The plasma etch completely consumes the alumina mask, leading to over-etching of the silicon nitride membrane. After etching, the thickness of the porous silicon nitride membrane is in the range of $50 \mathrm{~nm}$, which is less than its starting thickness of $100 \mathrm{~nm}$. As we discuss below, the self-assembled block copolymer film determines the pore density and initial porosity of the resulting nitride membrane. However, we can further tune the membrane's average pore size and porosity by conformally coating the structure with alumina by ALD (e.g., Figure 1e) - a useful modification process for enhancing the membranes selectivity to different gases. In a hexagonal arrangement of pores, the membrane porosity $(\eta)$ is given by: 


$$
\eta=\frac{\pi}{2 \sqrt{3}}\left(\frac{d}{\ell}\right)^{2}
$$

so that $\eta$ can vary between 0 (for $d=0$ ) and around 23\% (for $d \sim \ell / 2$ ).

Cross-sectional transmission electron microscope (TEM) images of completed membranes show a highly porous silicon nitride layer measuring thickness of $b=44 \pm 3 \mathrm{~nm}$ (Figure $2 \mathrm{a}$ ). Due to the difficulty of preparing cross sectional TEM specimens on suspended structures, the images in Figure 2 are taken on similarly processed silicon nitride films supported on silicon substrates. The tapered pore geometry flares to a diameter $(d)$ of more than $30 \mathrm{~nm}$ at the membrane top surface and narrows uniformly to an average diameter of $21 \mathrm{~nm}$ at the membrane bottom. The average pore size is reduced to $13 \mathrm{~nm}$ and $9 \mathrm{~nm}$ by ALD alumina coating of $4 \mathrm{~nm}$ and $6 \mathrm{~nm}$ thicknesses, respectively. Correspondingly, the membrane porosity is lowered to $9 \%$ and $4 \%$, respectively. The effectiveness of ALD alumina coating is verified by cross-sectional TEM imaging (Figure $2 \mathrm{~b}$ ), which also reveals that the pores are straight through, corresponding to unity tortuosity in the membrane.

We measure gaseous diffusion through these highly porous silicon nitride structures and quantitatively describe the behavior using a phenomenological transport model. Experimentally, we affix the membrane to the entry of a small chamber with volume $V_{1}=0.41 \mathrm{~cm}^{3}$, whose exit is connected to a residual gas analyzer (RGA) by a $50 \mu \mathrm{m}$ diameter, $50 \mathrm{~cm}$ length capillary (Figure 3a). The RGA chamber (with volume $V_{2} \gg V_{1}$ ) is continuously pumped with a turbomolecular pump. Exposing the membrane to a gas concentration $N_{0}$ results in gas transport across the membrane and gas concentration rises $N_{1}(t), N_{2}(t)$ in the chamber $\left(V_{1}\right)$ and RGA $\left(V_{2}\right)$, respectively. Upon exposing the attached membranes to saturated gaseous vapors of acetone (Figure 3b, black curve) and ethyl acetate (Figure 3b, red curve) under ambient conditions, we measure increases in their partial pressures within the RGA, $P(t) \propto N_{2}(t)$, with a 
qualitatively slower turn-on at short times (Figure 3b, inset), and a more rapid increase at longer times, before reaching saturation after more than 2,000 s. The pressure versus time curves for acetone and ethyl acetate are qualitatively similar, although the pressure rise of ethyl acetate occurs more gradually. The origin of the time axis denotes the point when the membranes are brought in contact with the vapor.

The gas flux $(d n / d t)$ through the membrane and capillary tube are proportional to the concentration differences across the interfaces $(\Delta N)$, a relation known as Fick's First Law:

$$
\begin{aligned}
& \frac{d n_{1}}{d t}=k_{1}\left(N_{0}-N_{1}\right) \\
& \frac{d n_{2}}{d t}=k_{2}\left(N_{1}-N_{2}\right)
\end{aligned}
$$

where $k_{1}$ and $k_{2}$ are phenomenological diffusion constants across the membrane and through the capillary, respectively (Figure 3a). We designed our experiments so that $k_{1}$ far exceeds $k_{2}$ by connecting the two chambers with a long, thin capillary tube and restricts flow. The coupled differential equations (2) have the solution:

$$
N_{2}(t)=N_{2}^{\infty}\left\{1-\frac{\beta}{\beta-\alpha} e^{-\alpha t}+\frac{\alpha}{\beta-\alpha} e^{-\beta t}\right\}
$$

in the limit of our experimental setup where $N_{2} \ll N_{1}$. In Eq. (3), the rate constants $\alpha$ and $\beta$ are associated with gas transport into and out of volumes $V_{1}$ and $V_{2}$, and are defined as $\alpha \equiv$ $\left(k_{1}+k_{2}\right) / V_{1} \approx k_{1} / V_{1}$, and $\beta \equiv\left(k_{2}+k_{3}\right) / V_{2}$, where $k_{3}$ is the pumping rate of the turbomolecular pump. $\quad N_{1}^{\infty}$ and $N_{2}^{\infty}$ are the steady state gas concentrations given by $N_{1}^{\infty} \equiv$ $k_{1} N_{0} /\left(k_{1}+k_{2}\right) \cong N_{0}$ and $N_{2}^{\infty} \equiv k_{2} N_{1}^{\infty} /\left(k_{2}+k_{3}\right) \cong k_{2} N_{0} /\left(k_{2}+k_{3}\right)$

At short times $\left(t<\alpha^{-1}, \beta^{-1}\right)$, the pressure rise in the RGA chamber, given by Eq. (3), reduces to

$$
N_{2}(t)=N_{2}^{\infty} \cdot \alpha \beta t^{2} / 2
$$


using a second order Taylor expansion. This quadratic time dependence is observed experimentally for $t<50 \mathrm{~s}$ (Figure 3b, inset). A plot of measured $\sqrt{P / P^{\infty}} \equiv \sqrt{N_{2} / N_{2}^{\infty}}$ versus $t$ for acetone flowing through silicon nitride membranes with different average pore sizes (i.e., porosities) shows that gas diffusion clearly slows in structures with smaller pores (Figure 4a). We use Eqs. (3) and (4) to determine the membrane's phenomenological diffusion constant $k_{1} \cong V_{1} \alpha$ for acetone, improving the accuracy of our analysis by first independently determining $\beta$ for our experimental setup by measuring the RGA pressure rise versus time without any membrane present. In this case, Eq. (3) reduces to $N_{2}(t) \propto\left(1-e^{-\beta t}\right)$ and we determine $\beta=(1.11 \pm 0.07) \times 10^{-2} \mathrm{~s}^{-1}$

The phenomenological diffusion constants $k_{l}$ are related to gas diffusivities within a single membrane pore $\left(D_{\mathrm{A}}\right)$ by Fick's First Law:

$$
\mathbf{J}=-D(\mathbf{r}) \cdot \nabla N(\mathbf{r})
$$

where $D(\mathbf{r})$ is the diffusivity at location $\mathbf{r}$ and equals the free space diffusivity $D_{0}$ outside the membrane. Within the membrane pore, $D(\mathbf{r})$ becomes $D_{\text {eff }}=\eta D_{\mathrm{A}} / \tau$, where $\eta$ is the porosity and $\tau=1$ the pore tortuosity. In order to correctly account for the complexity of our experimental geometry (Figure 5a), we numerically solve Eq. (5) by the finite element method using the $\mathrm{COMSOL}^{\mathrm{TM}}$ Multiphysics dilute gas transport module. From the solution, we calculate the gas flow through the membrane as $d n / d t=\iint_{S} \mathbf{J} \cdot d \mathbf{S}$ and the membrane diffusion constant as $k_{1}=\Delta N^{-1} d n / d t$, for a given gas concentration difference $\Delta N$ between the two sides and a given gas diffusivity profile $D(\mathbf{r})$. We experimentally measure the free space value of $D_{0}$ by removing the silicon nitride membrane from the windows, so that $D(\mathbf{r})=D_{0}$ across the entire space. For acetone, measured diffusion constant of $k_{1}=1.38 \times 10^{-3} \mathrm{~cm}^{3} \mathrm{~s}^{-1}$ (Figure $3 \mathrm{~b}$ ) corresponds to a calculated free-space diffusivity of $D_{0}^{\text {ace }}=0.139 \mathrm{~cm}^{2} \mathrm{~s}^{-1}$ (Supplementary Figure S1), which agrees well with the literature value of $0.124 \mathrm{~cm}^{2} \mathrm{~s}^{-1} \cdot{ }^{33}$ Our simulations 
provide a spatial profile of the acetone vapor flux in the vicinity of the open window (Figure $5 \mathrm{c}$ ). Similarly, we measure diffusion constants of $7.93 \times 10^{-4} \mathrm{~cm}^{3} \mathrm{~s}^{-1}$ for ethyl acetate vapor diffusing through the open window, which corresponds to a calculated free-space diffusivity $D_{0}^{\text {ea }}=0.080 \mathrm{~cm}^{2} \mathrm{~s}^{-1}$ - also closely matching the reported value of $0.073 \mathrm{~cm}^{2} \mathrm{~s}^{-1} .^{33}$

Systematically shrinking the membrane average pore diameter from its initial $21 \mathrm{~nm}$ to single digit nanometer dimensions slows the transport of acetone across the interface. Exposing membranes with smaller pore sizes and lower porosity to acetone vapor results in systematically slower initial pressure rises with time, consistent with reduced transport (Figure 4a). Membranes coated with alumina films thicker than $\geq 8 \mathrm{~nm}$ do not transport any gas, and appear completely blocked. We determine $k_{l}$ for different membranes from the linear slopes in Figure 4a, finding membrane diffusion constants ranging between $k_{1}=1.38 \times 10^{-3} \mathrm{~cm}^{3} \mathrm{~s}^{-1}$ for open windows and decreasing by an order of magnitude to $k_{1}=4 \times 10^{-4} \mathrm{~cm}^{3} \mathrm{~s}^{-1}$ in membranes with the smallest average pore sizes of $9 \mathrm{~nm}$ (Figure $4 \mathrm{~b})$.

The nanoporous, silicon nitride membranes reduce acetone diffusion to less than its free space value $D_{0}^{\text {ace }}$, with porous structures having $21 \mathrm{~nm}, 13 \mathrm{~nm}$, and $9 \mathrm{~nm}$ average pore diameters (Figure $4 \mathrm{~b}$ ) yielding corresponding effective diffusivities $D_{\text {eff }}=3.07 \times 10^{-4}, 1.13 \times 10^{-4}$, and $0.376 \times 10^{-4} \mathrm{~cm}^{2} \mathrm{~s}^{-1}$. Correcting these values for the different membrane porosities $(\eta)$ gives acetone diffusivities within the membrane pores $D_{\mathrm{A}}^{\text {ace }}=1.35 \times 10^{-3}, 1.30 \times 10^{-3}$, and $0.90 \times 10^{-3}$ $\mathrm{cm}^{2} \mathrm{~s}^{-1}$, respectively (Figure 6a). Similar measurements on ethyl acetate vapor flowing through these same membranes yield diffusivities of $D_{\mathrm{A}}^{\mathrm{ea}}=5.68 \times 10^{-4}, 3.74 \times 10^{-4}$, and $1.27 \times 10^{-4}$ $\mathrm{cm}^{2} \mathrm{~s}^{-1}$ within successively smaller pore diameters (Figure $6 \mathrm{~b}$ ). The ethyl acetate diffusivity is influenced more significantly by different pore sizes, which results in higher selectivity $\left(D_{\mathrm{A}}^{\mathrm{ace}} / D_{\mathrm{A}}^{\mathrm{ea}}\right)$ between the two gases in membranes with smaller pore sizes (Figure 6c).

These measured diffusion constants provide information about the mechanism of gas transport through the membrane. The membrane average pore diameter $d<20 \mathrm{~nm}$ and thickness 
$h \sim 50 \mathrm{~nm}$ are both smaller than the mean free path of gaseous molecules $(\lambda)$ at ambient pressure, which is estimated as $\sim 60 \mathrm{~nm}$ using

$$
\lambda=\frac{k_{B} T}{\sqrt{2} \pi a^{2} P}
$$

where $a \sim 4 \AA$ is the collision diameter, $P$ the pressure $\left(10^{5} \mathrm{~Pa}\right)$, and $T$ the temperature $(300 \mathrm{~K})$. Transport across thicker membranes with small pore sizes $(\lambda \gg d$ and $d \ll h)$ is generally governed by Knudsen diffusion, where the diffusivity scales inversely with the square root of the gas molecular weight $\left(M\left[\mathrm{~kg} \mathrm{~mol}^{-1}\right]\right)$,

$$
D_{K}=\frac{d}{3} \sqrt{\frac{8 R T}{\pi M}}
$$

with $R$ the gas constant. ${ }^{34}$ Eq. (7) estimates $D_{K} \sim 2.2 \times 10^{-2} \mathrm{~cm}^{2} \mathrm{~s}^{-1}$ for acetone through membranes with $d=21 \mathrm{~nm}$, which is over one order of magnitude larger than the measured values in our structures (Figure 6a). In addition, Eq. (7) predicts a membrane selectivity ratio between acetone and ethyl acetate of $D_{\mathrm{A}}^{\mathrm{ace}} / D_{\mathrm{A}}^{\mathrm{ea}}=\sqrt{M_{\mathrm{ea}} / M_{\mathrm{ace}}}=1.23$, which is similar to the free space selectivity $\left(D_{0}^{\text {ace }} / D_{0}^{\text {ea }}=1.74\right)$ and is independent of $d$. The measured values for the membranes however are much larger and depend strongly on $d$ (Figure 6c). The ideal selectivity, i.e., that determined from measuring pure vapors of acetone and ethyl acetate, is 2.4 for the largest pore size $(21 \mathrm{~nm})$ and increases to 7.1 for the smallest pore size $(9 \mathrm{~nm})$. The deviation from Knudsen diffusion and improved selectivity suggest that membrane transport is governed by a different mechanism.

Given the involvement of saturated vapors of acetone and ethyl acetate, we believe that the deviation is likely due to the capillary condensation of vapors within the membrane pores, which leads to (partial) blocking of the pores and improves the selectivity. ${ }^{34-36}$ Capillary condensation arises from the suppression of saturated vapor pressure for a liquid confined in a small volume 
such as a capillary, when the chemical potential of the condensed (liquid) phase is lowered by its surface energy. Within a capillary of diameter $d$, the suppressed vapor pressure $P$ is given by the Kelvin equation

$$
\frac{R T}{V_{\mathrm{m}}} \ln \frac{P}{P_{\mathrm{s}}}=-\frac{4 \sigma_{\mathrm{s}} \cos \theta}{d}
$$

where $P_{\mathrm{S}}$ is the bulk vapor pressure, $V_{\mathrm{m}}$ the liquid molar volume, $\sigma_{\mathrm{s}}$ the surface tension, and $\theta$ the contact angle. At $25^{\circ} \mathrm{C}$, the constants are $P_{\mathrm{s}}=31.9 \mathrm{kPa}, V_{\mathrm{m}}=73.4 \mathrm{~cm}^{3} \mathrm{~mol}^{-1}$, and $\sigma_{\mathrm{s}}=22.72 \mathrm{mN} \mathrm{m}^{-1}$ for acetone, and $12.1 \mathrm{kPa}, 97.7 \mathrm{~cm}^{3} \mathrm{~mol}^{-1}$, and $23.39 \mathrm{mN} \mathrm{m}^{-1}$ for ethyl acetate. $^{37}$ Since silicon nitride is wet perfectly by acetone and ethyl acetate, we take $\theta=0$ for both cases. As shown in Figure 6d, the vapor pressures of acetone and ethyl acetate are gradually suppressed as the pore diameter is narrowed below $20 \mathrm{~nm}$. Therefore, saturated vapors entering the pores become supersaturated and can condense within the pores to partially block the vapor transportation. Diffusion into volume $V_{1}$ must now rely on the re-evaporation of the trapped liquid droplets, which leads to the observed lower diffusivities. Due to its lower bulk vapor pressure $(\sim 1 / 3$ of acetone), liquid droplets of ethyl acetate evaporates much slower than those of acetone, thus contributing to higher selectivity ratio $D_{\mathrm{A}}^{\text {ace }} / D_{\mathrm{A}}^{\mathrm{ea}}$. The ideal selectivity is further improved when the pore narrows, as ethyl acetate experiences more significant capillary condensation than acetone, which is evident from the rising value of $P_{\text {ace }} / P_{\text {ea }}$ toward smaller pore diameters (Figure 6d).

In summary, we have leveraged high density of regular, nanoscale features in selfassembled block copolymer thin films to create ultrathin porous silicon nitride membranes suitable for gas transport. The inorganic membrane features high porosity and straight-through pores with predetermined size, which are ideal for high throughput transport. Conformal coating the structure with alumina shrinks membrane pore diameters from their initial $\sim 20 \mathrm{~nm}$ values to single-digit nanometer dimensions, a size range suitable for gas molecule selection based on 
Knudsen diffusion. In our studies, condensation of gaseous vapor within membrane pores drives the transport from the Knudsen regime and further increases selectivity between species. As a demonstration, we have achieved selectivities as high as 7:1 between vapors of acetone and ethyl acetate at ambient pressure and temperature.

\section{METHODS}

\section{Block copolymer (BCP) template fabrication}

Thin film PS- $b$-PMMA (71 kg mol ${ }^{-1}, 30: 70$ PS:PMMA in weight) is coated over the suspended silicon nitride window by spin casting from a toluene solution of the polymer (1\% w/v). Before applying the BCP film, the substrate is functionalized with a PS-r-PMMA-OH random copolymer neutral brush to ensure that the PS microdomains orient perpendicularly to the substrate. ${ }^{38}$ The film is annealed at $200^{\circ} \mathrm{C}$ for 4 hours to facilitate the self-assembly of an ordered cylindrical phase morphology of hexagonally arranged PS cylinders embedded in a PMMA matrix.

\section{Pattern transfer to silicon nitride membrane}

PMMA regions of the self-assembled polymer film is first selectively infused with alumina through six sequential exposures to vapor pressures of trimethylaluminum (>10 Torr, $5 \mathrm{~m}$ ) and water vapor $(\sim 10$ Torr, $5 \mathrm{~m})$ at $85{ }^{\circ} \mathrm{C}$, using a commercial ALD tool (Cambridge Nanotech Savannah S-100). The residue block copolymer is then removed using an oxygen plasma (20 W rf, $100 \mathrm{mTorr}, 3 \mathrm{~m}$ ), to leave a rugged mask of alumina that resembles the morphology of the original PMMA domains. The membrane is then placed in an inductively coupled plasma etcher (Oxford Instrument Plasmalab 100) for pattern transfer from the alumina mask to the silicon nitride membrane, using constant etching parameters of $10 \mathrm{sccm} \mathrm{O}_{2}, 100 \mathrm{sccm} \mathrm{CF}_{4}, 10 \mathrm{mTorr}$ pressure, $200 \mathrm{~W}$ RIE power, and $20{ }^{\circ} \mathrm{C}$ temperature for $90 \mathrm{~s}$. 
The as-fabricated pores are narrowed by conformally coating the porous membrane with ALD alumina (Cambridge Nanotech Savannah S-100). The process uses trimethylaluminum and water as the precursors and is carried out at $200{ }^{\circ} \mathrm{C}$, under 300 mTorr of nitrogen carrier gas flowing at $20 \mathrm{sccm}$. The nominal deposition rate is $0.1 \mathrm{~nm} /$ cycle.

\section{ASSOCIATED CONTENT}

\section{Supporting Information}

The Supporting Information is available free of charge on the ACS Publications website.

Simulation result for the diffusion constant versus free space diffusivity for an open membrane window.

\section{AUTHOR INFORMATION}

\section{Corresponding Authors}

* E-mail: mzliu@bnl.gov; ctblack@bnl.gov

${ }^{\dagger}$ Present address: Indian Institute of Science Education and Research, Pune, Maharashtra 411008, India

\section{ACKNOWLEDGMENT}

This research used resources of the Center for Functional Nanomaterials, which is a U.S. DOE Office of Science User Facility, at Brookhaven National Laboratory under Contract No. DE-SC0012704.

\section{REFERENCES}

1. Tan, X.; Li, K., Inorganic Membrane Reactors: Fundamentals and Applications. John Wiley \& Sons, Ltd: 2014. 
2. $\quad$ Arora, P.; Zhang, Z., Battery Separators. Chem. Rev. 2004, 104, 4419-4462.

3. Pendergast, M. M.; Hoek, E. M. V., A review of water treatment membrane nanotechnologies. Energy Environ. Sci. 2011, 4, 1946-1971.

4. Strathmann, H.; Grabowski, A.; Eigenberger, G., Ion-Exchange Membranes in the Chemical Process Industry. Ind. Eng. Chem. Res. 2013, 52, 10364-10379.

5. Yampolskii, Y.; Freeman, B., Membrane gas separation. Wiley: Hoboken, NJ, 2010.

6. Feng, X.; Huang, R. Y. M., Liquid Separation by Membrane Pervaporation: A Review. Ind. Eng. Chem. Res. 1997, 36, 1048-1066.

7. Shao, P.; Huang, R. Y. M., Polymeric membrane pervaporation. J. Membr. Sci. 2007, 287, 162-179.

8. Furneaux, R. C.; Rigby, W. R.; Davidson, A. P., The formation of controlled-porosity membranes from anodically oxidized aluminum. Nature 1989, 337, 147-149.

9. Bischoff, B. L.; Anderson, M. A., Peptization process in the sol-gel preparation of porous anatase $\left(\mathrm{TiO}_{2}\right)$. Chem. Mater. 1995, 7, 1772-1778.

10. Chang, C. H.; Gopalan, R.; Lin, Y. S., A comparative study on thermal and hydrothermal stability of alumina, titania and zirconia membranes. J. Membr. Sci. 1994, 91, 27-45.

11. Fukushima, M.; Zhou, Y.; Miyazaki, H.; Yoshizawa, Y. I.; Hirao, K.; Iwamoto, Y.; Yamazaki, S.; Nagano, T., Microstructural characterization of porous silicon carbide membrane support with and without alumina additive. J. Am. Ceram. Soc. 2006, 89, 1523-1529.

12. Cot, L.; Ayral, A.; Durand, J.; Guizard, C.; Hovnanian, N.; Julbe, A.; Larbot, A., Inorganic membranes and solid state sciences. Solid State Sci. 2000, 2, 313-334.

13. Burggraaf, A. J.; Cot, L., Fundamentals of inorganic membrane science and technology. Elsevier: Amsterdam ; New York, 1996.

14. Bates, F. S.; Fredrickson, G. H., Block copolymer thermodynamics - theory and experiment. Ann. Rev. Phys. Chem. 1990, 41, 525-557.

15. Ruiz, R.; Kang, H. M.; Detcheverry, F. A.; Dobisz, E.; Kercher, D. S.; Albrecht, T. R.; de Pablo, J. J.; Nealey, P. F., Density multiplication and improved lithography by directed block copolymer assembly. Science 2008, 321, 936-939.

16. Black, C. T.; Ruiz, R.; Breyta, G.; Cheng, J. Y.; Colburn, M. E.; Guarini, K. W.; Kim, H. C.; Zhang, Y., Polymer self assembly in semiconductor microelectronics. IBM J. Res. Dev. 2007, 51, 605-633.

17. Park, M.; Harrison, C.; Chaikin, P. M.; Register, R. A.; Adamson, D. H., Block Copolymer Lithography: Periodic Arrays of $\sim 10^{11}$ Holes in 1 Square Centimeter. Science 1997, 276, 1401-1404.

18. Liu, C. C.; Nealey, P. F.; Raub, A. K.; Hakeem, P. J.; Brueck, S. R. J.; Han, E.; Gopalan, P., Integration of block copolymer directed assembly with 193 immersion lithography. J. Vac. Sci. Tech. B 2010, 28, C6b30-C6b34.

19. Harrison, C.; Park, M.; Chaikin, P. M.; Register, R. A.; Adamson, D. H., Lithography with a mask of block copolymer microstructures. J. Vac. Sci. Tech. B 1998, 16, 544-552.

20. Phillip, W. A.; Rzayev, J.; Hillmyer, M. A.; Cussler, E. L., Gas and water liquid transport through nanoporous block copolymer membranes. J. Membr. Sci. 2006, 286, 144-152.

21. Phillip, W. A.; Amendt, M.; O'Neill, B.; Chen, L.; Hillmyer, M. A.; Cussler, E. L., Diffusion and Flow Across Nanoporous Polydicyclopentadiene-Based Membranes. ACS Appl. Mater. Interfaces 2009, 1, 472-480.

22. Phillip, W. A.; Martono, E.; Chen, L.; Hillmyer, M. A.; Cussler, E. L., Seeking an ammonia selective membrane based on nanostructured sulfonated block copolymers. J. Membr. Sci. 2009, 337, 39-46.

23. Jackson, E. A.; Hillmyer, M. A., Nanoporous Membranes Derived from Block Copolymers: From Drug Delivery to Water Filtration. ACS Nano 2010, 4, 3548-3553. 
24. Ahn, H.; Park, S.; Kim, S. W.; Yoo, P. J.; Ryu, D. Y.; Russell, T. P., Nanoporous Block Copolymer Membranes for Ultrafiltration: A Simple Approach to Size Tunability. ACS Nano 2014, 8, 11745-11752.

25. Black, C. T.; Guarini, K. W.; Breyta, G.; Colburn, M. C.; Ruiz, R.; Sandstrom, R. L.; Sikorski, E. M.; Zhang, Y., Highly porous silicon membrane fabrication using polymer selfassembly. J. Vac. Sci. Tech. B 2006, 24, 3188-3191.

26. Nunes, S. P.; Car, A., From Charge-Mosaic to Micelle Self-Assembly: Block Copolymer Membranes in the Last 40 Years. Ind. Eng. Chem. Res. 2013, 52, 993-1003.

27. Yang, S. Y.; Park, J.; Yoon, J.; Ree, M.; Jang, S. K.; Kim, J. K., Virus filtration membranes prepared from nanoporous block copolymers with good dimensional stability under high pressures and excellent solvent resistance. Adv. Funct. Mater. 2008, 18, 1371-1377.

28. Kim, J. K.; Yang, S. Y.; Lee, Y.; Kim, Y., Functional nanomaterials based on block copolymer self-assembly. Prog. Polym. Sci. 2010, 35, 1325-1349.

29. Ruiz, R.; Kang, H.; Detcheverry, F. A.; Dobisz, E.; Kercher, D. S.; Albrecht, T. R.; de Pablo, J. J.; Nealey, P. F., Density Multiplication and Improved Lithography by Directed Block Copolymer Assembly. Science 2008, 321, 936-939.

30. Rahman, A.; Liu, M. Z.; Black, C. T., Block copolymer self assembly for design and vapor-phase synthesis of nanostructured antireflective surfaces. J. Vac. Sci. Tech. B 2014, 32, 06FE02.

31. Peng, Q.; Tseng, Y.-C.; Darling, S. B.; Elam, J. W., Nanoscopic Patterned Materials with Tunable Dimensions via Atomic Layer Deposition on Block Copolymers. Adv. Mater. 2010, 22, 5129-5133.

32. Ramanathan, M.; Tseng, Y.-C.; Ariga, K.; Darling, S. B., Emerging trends in metalcontaining block copolymers: synthesis, self-assembly, and nanomanufacturing applications. $J$. Mater. Chem. C 2013, 1, 2080-2091.

33. GSI Chemical Properties Database. GSI Environmental Inc.: 2011.

34. Tan, X.; Li, K., Porous Membrane Reactors. In Inorganic Membrane Reactors, John Wiley \& Sons, Ltd: 2014; pp 27-73.

35. Ash, R.; Barrer, R. M.; Lowson, R. T., Transport of Single Gases and of Binary GasMixtures in a Microporous Carbon Membrane. J. Chem. Soc. Farad. Trans. 1 1973, 69, 21662178.

36. Sperry, D. P.; Falconer, J. L.; Noble, R. D., Methanol—hydrogen separation by capillary condensation in inorganic membranes. J. Membr. Sci. 1991, 60, 185-193.

37. Lide, D. R., CRC handbook of chemistry and physics. 89th ed.; CRC Press: Boca Raton, FL, 2008.

38. Mansky, P.; Liu, Y.; Huang, E.; Russell, T. P.; Hawker, C., Controlling Polymer-Surface Interactions with Random Copolymer Brushes. Science 1997, 275, 1458-1460. 

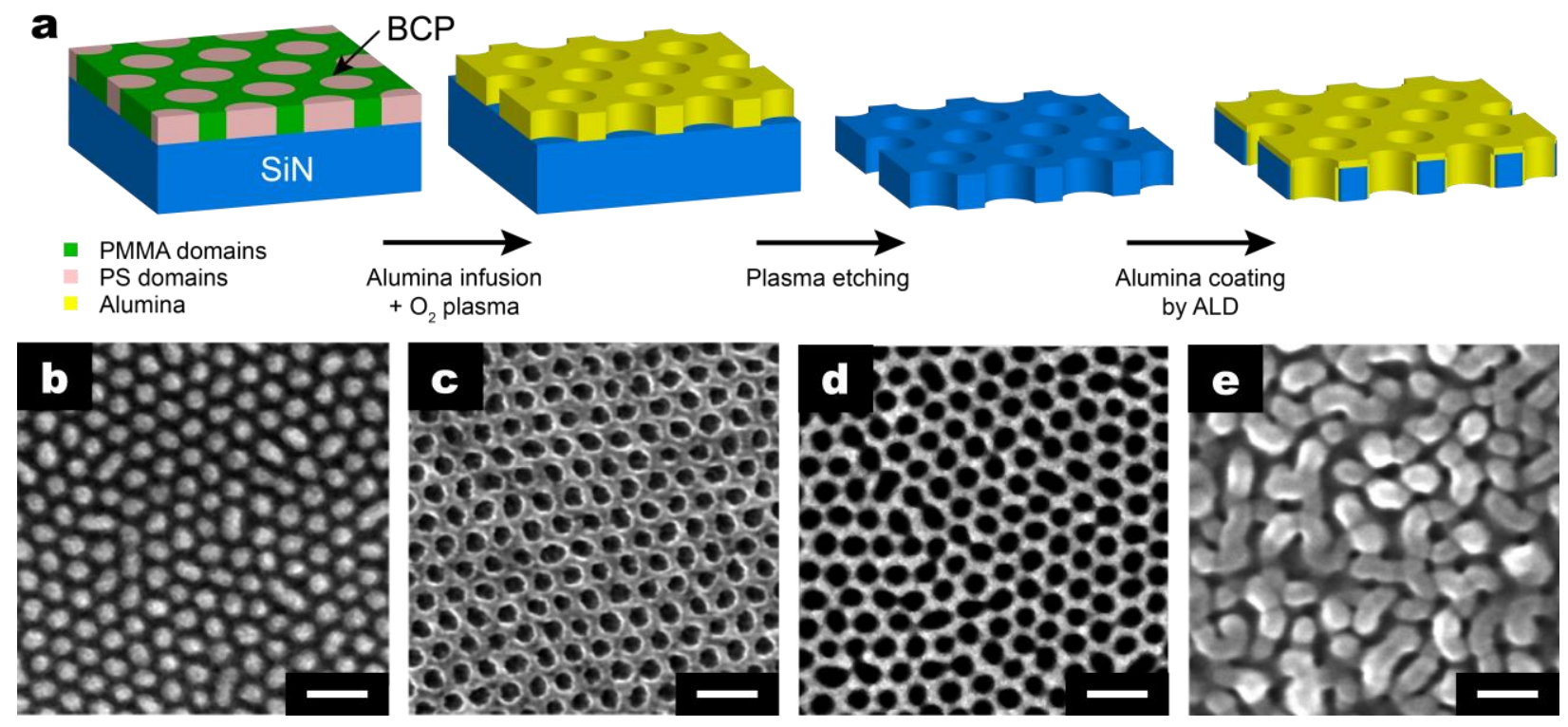

Figure 1. (a) Fabrication process for the nanoporous silicon nitride membranes from block copolymer templates. Scanning electron microscope images of (b) self assembled PS-b-PMMA cylindrical phase block copolymer thin film; (c) porous alumina thin film formed by block selective infiltration and polymer removal; (d) porous silicon nitride membrane; and (e) porous silicon nitride membrane conformally coated with $\sim 6 \mathrm{~nm}$ of alumina. Scale bar in all images denotes $100 \mathrm{~nm}$. 

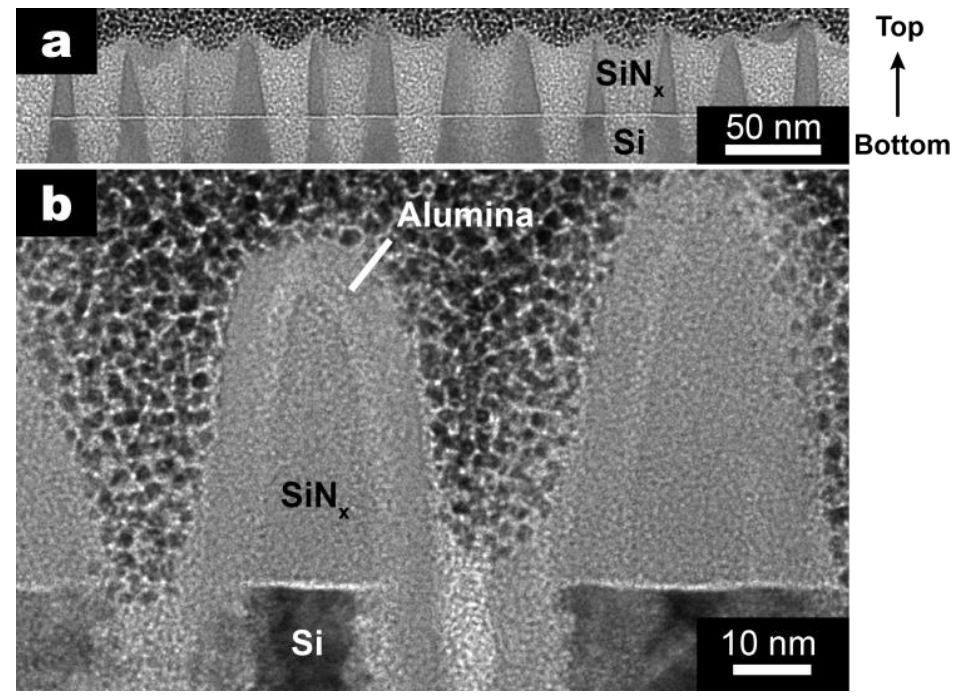

Figure 2. Cross-sectional transmission electron microscope images of (a) nanoporous silicon nitride film; (b) closeup of silicon nitride pores coated with $6 \mathrm{~nm}$ of alumina. Due to the difficulty of preparing cross sectional TEM specimens on suspended structures, the images are taken on similarly processed silicon nitride films supported on silicon substrates. 

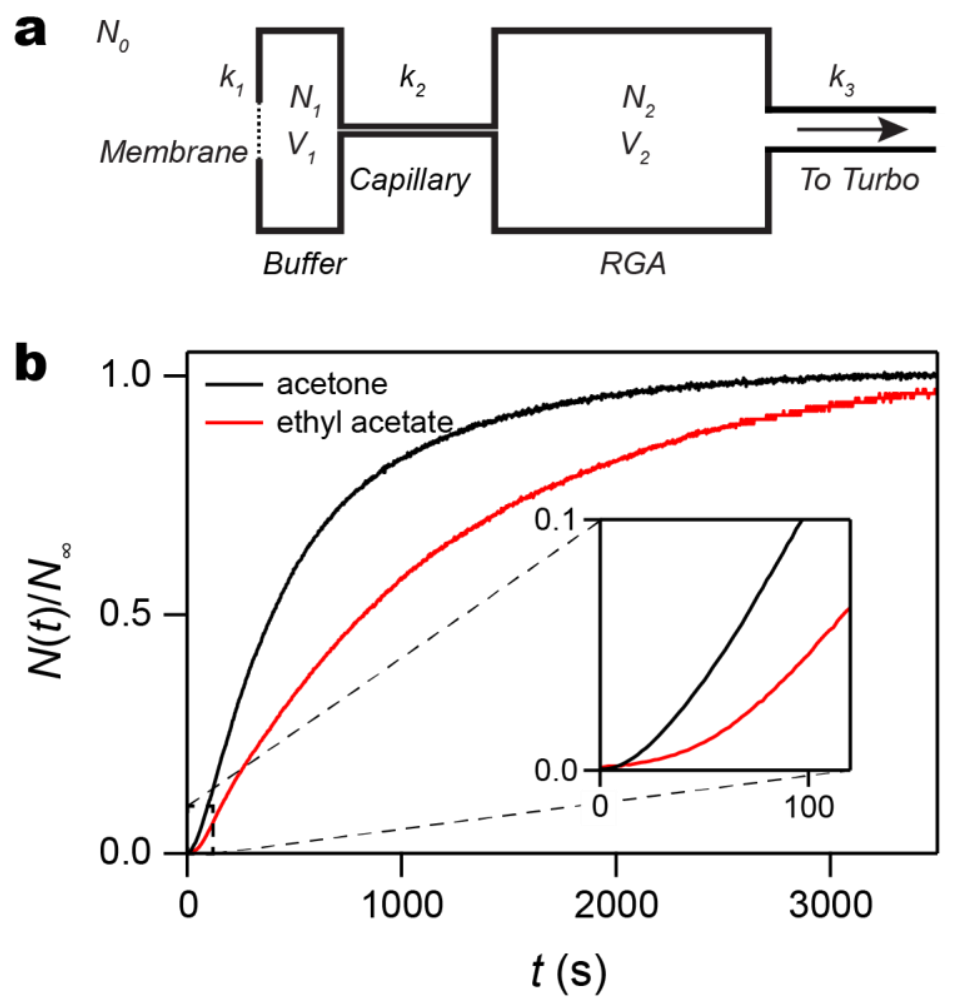

Figure 3. (a) Schematic experimental setup. (b) Normalized concentrations of acetone (black) and ethyl acetate (red) vapor versus time passing through the nanoporous membrane. (inset) At short times, the concentrations rise in proportion to (time) $)^{2}$. 

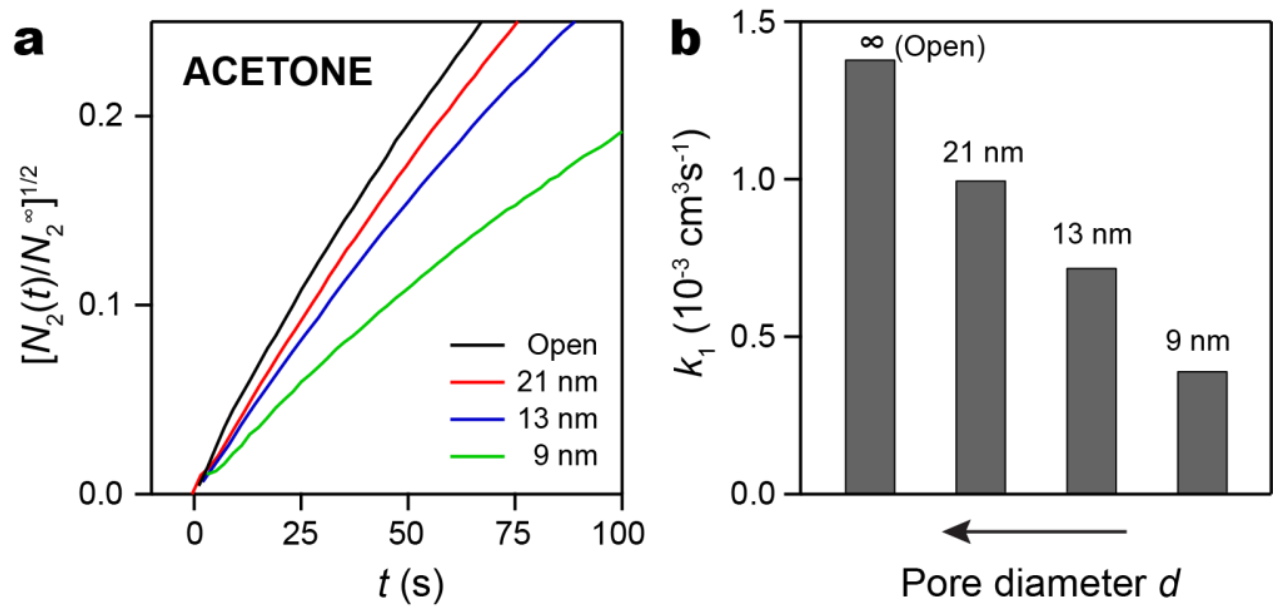

Figure 4. (a) The rate of acetone vapor diffusion through the membrane slows with decreasing pore size, being highest with an open aperture (black), followed by membranes with average pore diameters of $21 \mathrm{~nm}$ (uncoated nanoporous silicon nitride, red), $13 \mathrm{~nm}$ (4 nm alumina coating, blue), and $9 \mathrm{~nm}$ (6 $\mathrm{nm}$ alumina coating, green). (b) Acetone diffusion constant $k_{1}$ for membranes with different average pore sizes. 
a
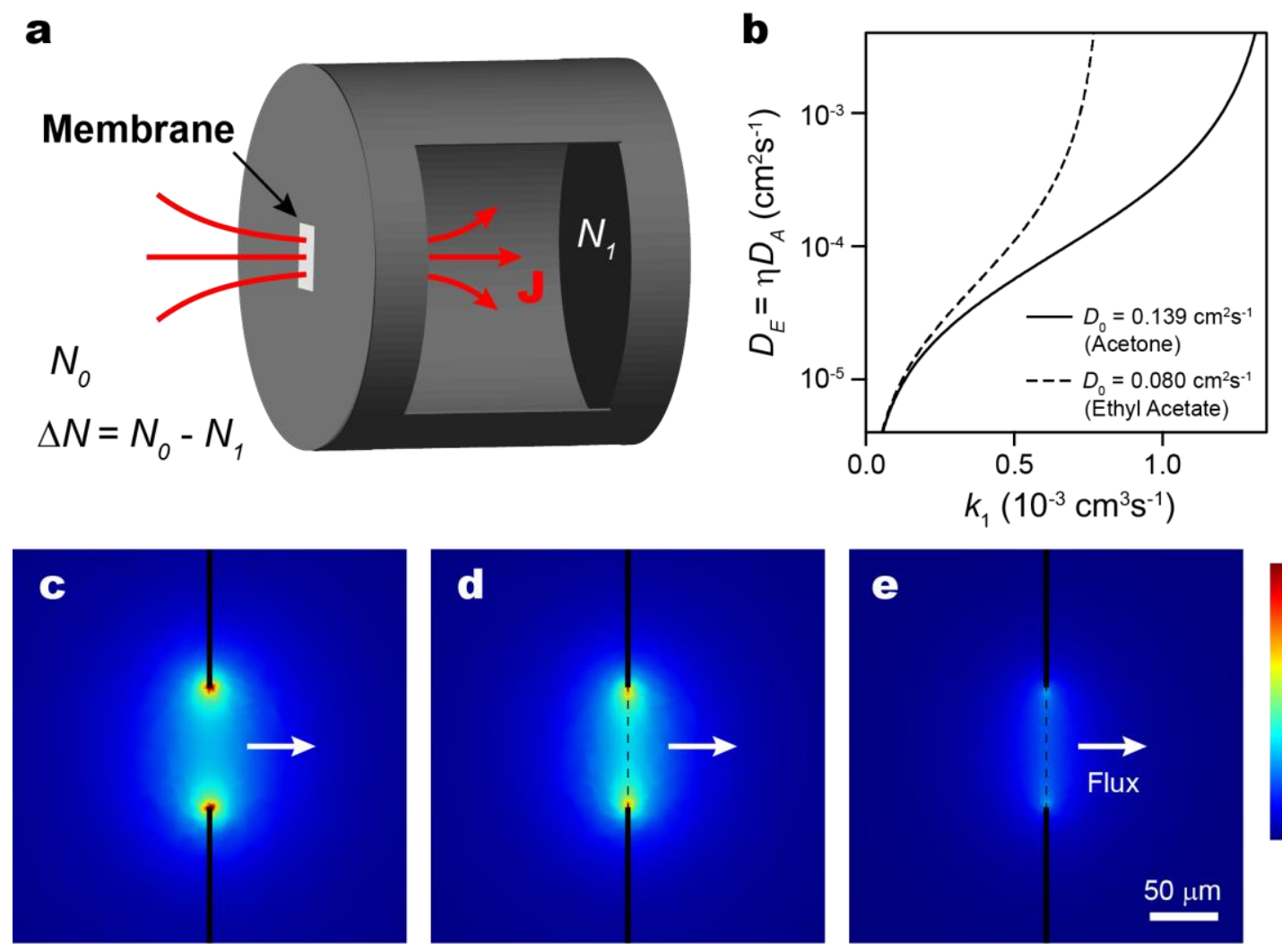

No membrane (Open) $D_{0}=0.139 \mathrm{~cm}^{2} \mathrm{~s}^{-1}$
$D_{E}=5 \times 10^{-4} \mathrm{~cm}^{2} \mathrm{~s}^{-1}$

$D_{0}=0.139 \mathrm{~cm}^{2} \mathrm{~s}^{-1}$

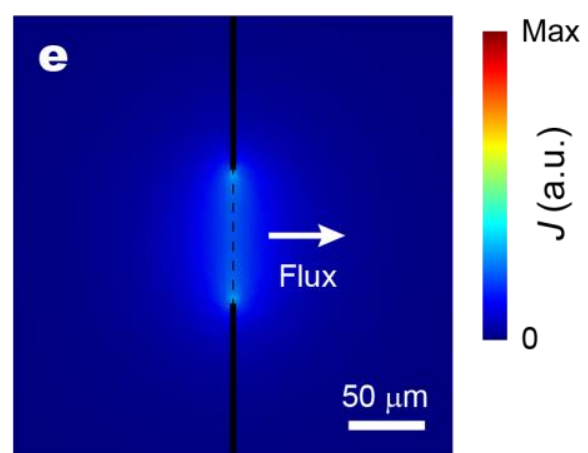

$D_{E}=5 \times 10^{-5} \mathrm{~cm}^{2} \mathrm{~s}^{-1}$

$D_{0}=0.139 \mathrm{~cm}^{2} \mathrm{~s}^{-1}$

Figure 5. (a) The finite element simulation models the membrane as a thin diffusion barrier and calculates steady state gas flux $\mathbf{J}(\mathbf{r})$ as a constant concentration difference $\Delta N=N_{0}-N_{1}$ is applied across the barrier. (b) Simulation results for the diffusion constant $k_{1}$ versus the membrane's effective diffusivity $D_{E}$, for acetone $\left(D_{0}=0.139 \mathrm{~cm}^{2} \mathrm{~s}^{-1}\right)$ and ethyl acetate $\left(D_{0}=\right.$ $0.080 \mathrm{~cm}^{2} \mathrm{~s}^{-1}$ ). The spatial profiles of flux in the vicinity of the membrane window are plotted in (c) for the case of open window and in (d) and (e) for membranes of various effective diffusivity $D_{E}$. 

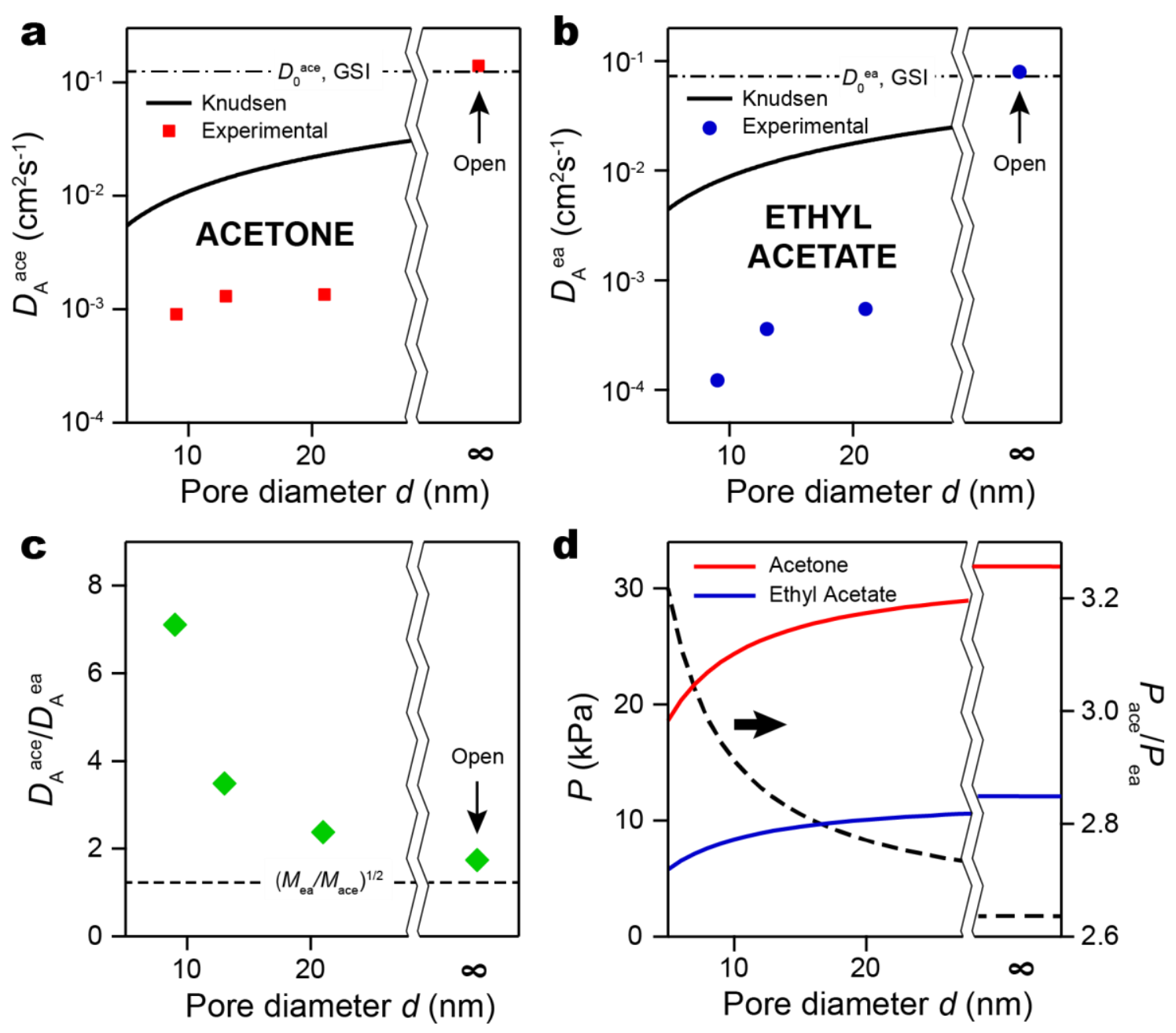

Figure 6. (a) The actual diffusivities of acetone (red squares) measured for various pore sizes are about one order of magnitude than the diffusivity expected from the Knudsen model (black line). For the case of open window, the measured diffusivity matches literature value very well. (b) Similar observation is made for ethyl acetate, except that at smaller pore size its diffusivity is suppressed more significantly. (c) A higher membrane selectivity $\left(D_{A}{ }^{a c e} / D_{A}{ }^{e a}\right)$ is observed at smaller membrane pore sizes. At the smallest pore size $(\sim 9 \mathrm{~nm})$ the selectivity is about 4 times more than Knudsen model's expectation $\left(\left(M_{e a} / M_{a c e}\right)^{1 / 2}\right)$. (d) The vapor pressures of acetone and ethyl acetate and their ratio $\left(P_{a c e} / P_{e a}\right)$ as functions of pore diameter, according to Eq. (8). 
TOC Figure
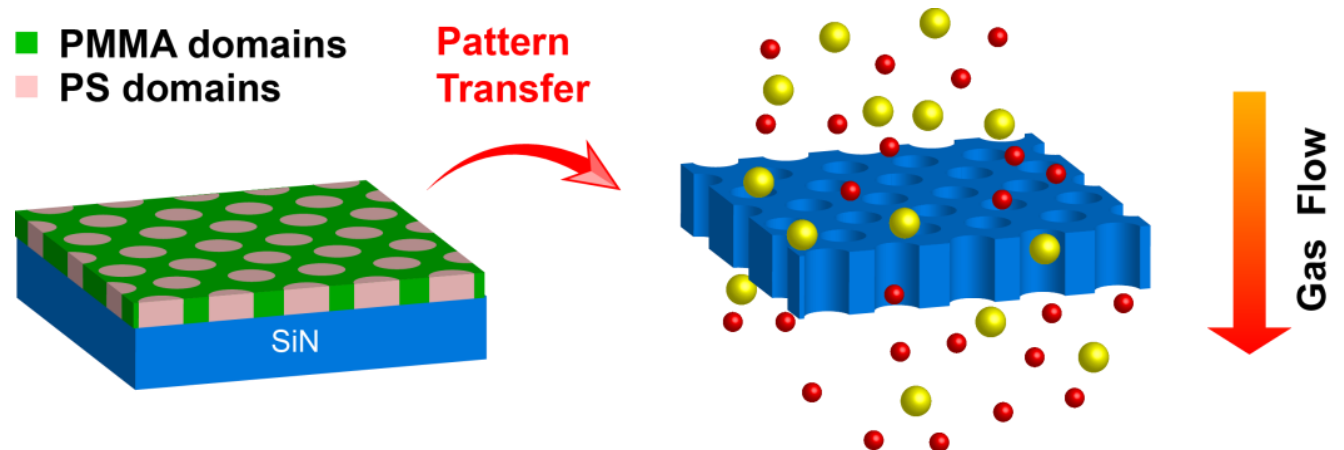

Block Copolymer Pattern Inorganic Porous Membrane 